\title{
Pascal et l'agonie du Christ à Gethsémani
}

\section{Philippe Sellier}

\section{OpenEdition}

Journals

Édition électronique

URL : http://journals.openedition.org/ccibp/1156

DOI : 10.4000/ccibp. 1156

ISSN : 2493-7460

\section{Éditeur}

Centre international Blaise Pasca

\section{Édition imprimée}

Date de publication : 15 décembre 2015

Pagination : 7-30

ISBN : 978-2-84516-671-4

ISSN : 0249-6674

\section{Référence électronique}

Philippe Sellier, «Pascal et l'agonie du Christ à Gethsémani », Courrier du Centre international Blaise Pascal [En ligne], 37 | 2015, mis en ligne le 16 mai 2018, consulté le 26 janvier 2021. URL : http:// journals.openedition.org/ccibp/1156 ; DOI : https://doi.org/10.4000/ccibp.1156

Ce document a été généré automatiquement le 26 janvier 2021.

Centre international Blaise Pascal 


\title{
Pascal et l'agonie du Christ à Gethsémani
}

\author{
Philippe Sellier
}

1 L'épisode déchirant de l'agonie du Christ au Mont des oliviers a hanté de nombreuses générations chrétiennes. Étrangement sa plus voyante apothéose littéraire se situe en France à l'époque romantique, où la solitude nocturne et l'abandon de Jésus correspondent à la sensibilité de l'époque : en 1844, Nerval publie « Le Christ au Mont des oliviers ", et Vigny «Le Mont des oliviers »; en 1855, le Jour des morts, Hugo désespéré par la mort de sa fille Léopoldine, grave puissamment la conception romantique au cœur du magnifique épilogue des Contemplations, « À celle qui est restée en France » :

Oh! quoi que nous fassions et quoi que nous disions,

Soit que notre âme plane au vent des visions,

Soit qu'elle se cramponne à l'argile natale,

Toujours nous arrivons à ta grotte fatale,

Gethsémani, qu'éclaire une vague lueur!

ô rocher de l'étrange et funèbre sueur !

2 La "grotte ", le « rocher » et la « vague lueur »- absents des évangiles - proviennent de gravures ou de tableaux comparables à la célèbre Prière dans les jardins de Mantegna (1460) ou Jésus au jardin des oliviers de Carpaccio (1502). Mais tous ces écrivains ne sont plus catholiques : leur religiosité floue dissout la foi chrétienne, et la disperse en petits conglomérats de souvenirs des scènes reçues dans l'enfance, lues dans la Bible ou vues dans les images.

3 Pour découvrir l'ardent saisissement qui caractérise la foi devant les paroles d'angoisse et la sueur de sang, il faut se tourner vers d'authentiques croyants, comme Péguy "épouvanté de ces lignes, de ces quelques lignes atroces, de ces paroles effrayantes, de cette effrayante prière "-Que ce calice passe loin de moi, si c'est possible - et de cette plainte : Mon âme est triste jusqu'à la mort. Péguy confondu devant le fait que « Dieu même a craint la mort ${ }^{1}$."

4 Le XVII ${ }^{\mathrm{e}}$ siècle avait vu auparavant se multiplier les appels à contempler la richesse théologique, et donc spirituelle, de cet épisode, ou - selon le lexique du cardinal de 
Bérulle - de ce «mystère" de la vie de Jésus. L'oratorien Charles de Condren, successeur de Bérulle, était même allé jusqu'à écrire :

La Passion de Jésus-Christ ne s'est pas faite seulement au Calvaire [...] il ne porte pas toutes sortes de peines en même temps [...] Mais elle s'est passée tout entière dans le Jardin des Olives dans l'esprit de Jésus. Je crois qu'il a plus souffert en ce mystère qu'en les autres endroits de sa Passion [...]

Le Sauveur du monde ne vous a pas seulement rachetée par la Croix et par les plaies qui ont percé son corps; les peines sacrées de son divin esprit; les agonies de son âme, les horreurs et les douleurs qu'il a voulu supporter de nos péchés, ont fait la plus digne partie de votre rédemption, et qui vous doit être en singulière dévotion [...]

[La Croix est] chose grande, mais de voir sa bénite âme en cet état d'extrême affliction, au milieu de tant et de si puissantes angoisses, toujours soucieuse de vous, plus pressée du souvenir de vos fautes [...], c'est un chef-d'œuvre de sa charité [...]

Soyez toujours dévote à la passion intérieure du Fils de Dieu, c'est-à-dire aux peines de son âme [...] Faites tous les mois une Communion en l'honneur de ces peines, et de la Justice divine qui les lui a imposées pour vous en délivrer².

Peu après Condren, le dominicain Louis Chardon compose des Méditations sur la Passion de Notre Seigneur Jésus-Christ pour tous les jours de l'année (1650), dont près de 130 concernent Gethsémani. Dans le sillage de Condren, Chardon pense que, sans les terribles souffrances intérieures de l'Agonie, toutes les autres souffrances de la Passion «n'eussent de rien servi pour la fin de notre rédemption ${ }^{3}$ ».

Mais l'inattendu surgit du fait que les textes les plus fascinants écrits sur l'Agonie proviennent d'un jeune mathématicien et physicien passé progressivement des sciences à la théologie, Blaise Pascal. Il est douteux que ce dernier ait lu Chardon - nous le verrons - mais il est incontestable que Condren a étendu sur lui son magistère spirituel et a laissé sur son œuvre religieuse une empreinte profonde ${ }^{4}$. Condren faisait de Gethsémani le sommet des souffrances de Jésus, Pascal lui emboîte le pas, et c'est pourquoi ce « mystère » brille d'un tel éclat dans ses œuvres.

7 Il s'impose de commencer par inventorier les textes où s'affirme la présence de l'Agonie. À partir de là, l'étude passera à l'approfondissement de la richesse du «mystère ». Dans la vision chrétienne de Dieu, de l'univers et de l'histoire, le mot «mystère » ne désigne nullement une énigme qui se dresse en face de la raison, mais une fenêtre ouverte sur l'infini, où la raison a la sagesse de se reconnaitre dépassée, inapte. Aux yeux du savant qu'est Pascal, la grandeur de la raison consiste à fonctionner pleinement dans son domaine - les sciences, les techniques et, de façon titubante, la recherche fragile d'une "prudence » dans la conduite de la vie - et à connaître ses limites: "Tout ce qui est incompréhensible ne laisse pas d'être.» (fr. 182).

\section{Les lieux de l'icône}

8 Icône centrale de la christologie pascalienne, Gethsémani apparaît dès octobre 1651 dans la riche lettre sur la mort d'Étienne Pascal. Au sein d'une mosaïque biblique se trouve rappelée la nécessité affirmée par le Christ lui-même qu'il passe par les souffrances pour entrer dans sa gloire (Luc 24, 26), et par le douloureux apprentissage de l'obéissance à en mourir (Philippiens 2, 6 et 8). Ce rappel est lié à l'épisode du Jardin des oliviers : «Au jour de sa chair, ayant crié avec un grand cri à celui qui pouvait le 
sauver de la mort, il a été exaucé pour sa révérence 5 . » Il s'agit d'une reprise de la Lettre aux Hébreux $(5,7)$ : «C'est lui [le Christ] qui, aux jours de sa [vie de] chair, offrit des prières et des supplications avec un cri puissant et des larmes à Celui qui pouvait le sauver de la mort en raison de sa piété ${ }^{6}$. La prière au Père de le sauver de la mort, si c'était possible, renvoie aux versets des évangiles synoptiques : «Père, s'il est possible, que passe loin de moi cette coupe.» Le "cri puissant» et les "larmes" - détails apportés par la Lettre, héritière ici d'une tradition plus précise que celle des synoptiques - correspondent au verset de Luc $(22,44)$ : «En proie à l'anxiété, il priait plus intensément ».

Quatre ans plus tard, en 1655, Pascal compose un Abrégé de la vie de Jésus-Christ, inspiré des quatre récits évangéliques, de deux travaux de Jansénius (Tetrateuchus et Series vitae Jesu Christi) et d'une "Concorde» d'Antoine Arnauld (Historia et concordia evangelica), mais avec une élaboration très originale. L'ouvrage est inachevé, bien que sa première rédaction ait conduit au terme de la « vie » de Jésus. Son auteur a visiblement pris peu à peu conscience de la valeur poétique et spirituelle des silences entre les articles, dont chacun est solennisé par la majuscule initiale, comme cela se fait en poésie classique. Ce qui explique que le récit se fragmente de plus en plus, à mesure qu'on avance. Or, de façon extrêmement nette, l'épisode de Gethsémani illustre la fréquence de cet appel au silence. C'est la première fois que le récit est autant fragmenté : une courte phrase est coupée en trois par des points et des blancs (211-213). Les cassures peuvent laisser en suspens des participes présents (205 et 207). Une telle caractéristique ne reparaîtra que pour la mort de Jésus (279-285), son ensevelissement (296-303) et sa résurrection (316-320). Tout se passe comme si une bonne partie de ces éclats de texte, allégés des références bibliques ou patristiques qui alourdissaient les deux livres de Jansénius, avaient été travaillés pour servir de support et d'élan à la méditation contemplative. Voici donc la séquence de l'Agonie :

205. Il sort de la maison pour aller au Mont des olives, et ayant passé le torrent du Cédron,

206. Il vint, en un jardin de Gethsémani,

207. Et laissant ses disciples, fut au mont des Olives à son ordinaire.

208. Il prend avec soi Pierre, Jacques et Jean, et étant en tristesse, leur dit que son âme est triste jusqu'à la mort.

209. Il s'éloigne un peu d'eux,

210. D'environ le jet d'une pierre.

211. Il prie.

212. La face en terre.

213. Trois fois.

214. À chaque fois, il vient à ses disciples et les trouve dormants.

215. L'ange le conforte (dans la destitution de toute consolation et divine et humaine, où sa nature humaine était réduite). Et dans cette agonie, il sue le sang.

216. Judas s'approche, et ses troupes.

217. Jésus les renverse tous d'une parole.

218. Judas le baise. Jésus se livre. Pierre coupe l'oreille de Malchus. Jésus l'en reprend.

219. Et le guérit.

220. Jésus, en se livrant, prie qu'on laisse aller les siens.

221. Jésus est amené, et les disciples s'enfuient. Et un jeune homme le suivant nu dans un drap, on le veut prendre. Il quitte son drap et s'enfuit nu? ${ }^{7}$.

Fait digne de remarque, alors que l'ensemble de l'Abrégé condense fortement les récits évangéliques, l'épisode de Gethsémani en occupe à lui seul 17 articles, pour les 20 versets de Matthieu ou de Marc, et les 12 de Luc. Une rare puissance de synthèse éclate 
pourtant dans la finesse du tissage des reprises : si Matthieu a en propre «la face en terre ", Marc est le seul à mentionner la fuite d'un jeune disciple tout nu. À Luc l'Abrégé emprunte les détails sur la distance « D'environ le jet d'une pierre », la sueur de sang, la consolation angélique et le mot capital d'« agonie ». Le terme « jardin » ne figurait que dans Jean $(18,1)$, mais son équivalent latin, «Hortus» servait de titre à une petite section de la Concorde d'Arnauld. La «destitution de toute consolation» provient du Tetrateuchus de Jansénius : "Quand la consolation (solatium) tant divine qu'humaine avait abandonné (destituerat) sa nature humaine.» (Sur Luc 22, 43). Ces quelques exemples suffisent à mettre en lumière le travail de l'intelligence pascalienne.

11 Si le genre littéraire des Écrits sur la grâce et celui des Provinciales, avec leur vivacité polémique, n'étaient guère favorables à une émergence de l'Agonie, celle-ci en revanche s'affirme dans les Pensées, rédigées entre la fin de 1656 et 1662. Il est difficile de savoir si dans la formule "Dieu humilié » (fr. 285, 299) Pascal entendait résonner l'étymologie (humus, la terre) et le « La face en terre » de l'Agonie. Mais des occurrences explicites du récit de Gethsémani apparaissent dans les fr. 460 (Jésus et Judas), et 461 : "Priez de peur d'entrer en tentation [Luc 22, 40 et 46] », puis l'allusion au geste de Pierre à l'encontre de Malchus (Luc 22, 48-41].

De façon beaucoup plus étendue, l'apologiste s'empresse de tirer parti du contraste mystérieux qui oppose un Jésus dans la détresse à la personnalité toute-puissante qui avance d'un pas décidé vers sa Passion et renverse les gardes venus pour l'arrêter (Jean $18,6)$ :

Qui a appris aux évangélistes les qualités d'une âme parfaitement hérö̈que pour la peindre si parfaitement en Jésus-Christ? Pourquoi le font-ils faible dans son agonie ? Ne savent-ils pas peindre une mort constante ? Oui. Car le même saint Luc peint celle de saint Étienne [Actes des apôtres 7, 58-59] plus forte que celle de JésusChrist.

Ils le font donc capable de crainte, avant que la nécessité de mourir soit arrivée, et ensuite tout fort.

Mais quand ils le font si troublé, c'est quand il se trouble lui-même. Et quand les hommes le troublent, il est tout fort. (fr.347)

Ce fragment a été écrit au printemps de 1658, comme l'atteste le filigrane du papier qui le porte, selon les conclusions de Pol Ernst dans son étude de 1996 Les Pensées de Pascal Géologie et stratigraphie. Il annonce ou rappelle le magnifique poème en prose intitulé judicieusement «Le Mystère de Jésus » (on ignore par qui), et sur lequel il nous faut nous arrêter maintenant :

Le Mystère de Jésus

Jésus souffre dans sa Passion les tourments que lui font les hommes. Mais dans l'agonie il souffre les tourments qu'il se donne à lui-même. Turbare semetipsum. C'est un supplice d'une main non humaine, mais toute-puissante. Et il faut être toutpuissant pour le soutenir.

Jésus cherche quelque consolation au moins dans ses trois plus chers amis, et ils dorment. Il les prie de soutenir un peu avec lui, et ils le laissent avec une négligence entière, ayant si peu de compassion qu'elle ne pouvait seulement les empêcher de dormir un moment. Et ainsi Jésus était délaissé seul à la colère de Dieu.

Jésus est seul dans la terre non seulement qui ressente et partage sa peine, mais qui la sache. Le ciel et lui sont seuls dans cette connaissance. 
Jésus est dans un jardin, non de délices, comme le premier Adam, où il se perdit et tout le genre humain, mais dans un de supplices, où il s'est sauvé et tout le genre humain.

Il souffre cette peine et cet abandon dans l'horreur de la nuit.

Je crois que Jésus ne s'est jamais plaint que cette seule fois. Mais alors il se plaint comme s'il n'eût plus pu contenir sa douleur excessive : Mon âme est triste jusqu'à la mort.

Jésus cherche de la compagnie et du soulagement de la part des hommes. Cela est unique en toute sa vie, ce me semble. Mais il n'en reçoit point, car ses disciples dorment.

Jésus sera en agonie jusqu'à la fin du monde. Il ne faut pas dormir pendant ce temps-là.

Jésus au milieu de ce délaissement universel et de ses amis choisis pour veiller avec lui, les trouvant dormant, s'en fâche à cause du péril où ils exposent non lui, mais eux-mêmes, et les avertit de leur propre salut et de leur bien avec une tendresse cordiale pour eux pendant leur ingratitude, et les avertit que l'esprit est prompt et la chair infirme.

Jésus, les trouvant encore dormant, sans que ni sa considération ni la leur les en eût retenus, il a la bonté de ne pas les éveiller, et les laisse dans leur repos.

Jésus prie dans l'incertitude de la volonté du Père, et craint la mort. Mais l'ayant connue, il va au-devant s'offrir à elle : Eamus. Processit (Joannes).

Jésus a prié les hommes, et n'en a pas été exaucé.

Jésus, pendant que ses disciples dormaient, a opéré leur salut.

Il l'a fait à chacun des justes pendant qu'ils dormaient et dans le néant avant leur naissance, et dans les péchés depuis leur naissance.

Il ne prie qu'une fois que le calice passe, et encore avec soumission, et deux fois qu'il vienne, s'il le faut.

Jésus dans l'ennui.

Jésus, voyant tous ses amis endormis, et tous ses ennemis vigilants, se remet tout entier à son Père.

Jésus ne regarde pas dans Judas son inimitié, mais l'ordre de Dieu qu'il aime, et la voit si peu qu'il l'appelle ami.

Jésus s'arrache d'avec ses disciples pour entrer dans l'agonie. Il faut s'arracher de ses plus proches et des plus intimes pour l'imiter.

Jésus étant dans l'agonie et dans les plus grandes peines, prions plus longtemps.

Ici prend fin cette fervente contemplation du Christ à Gethsémani. La perfection de son unité littéraire saute aux yeux, soulignée par la reprise du nom de Jésus, qui ouvre dixsept des vingt versets, remplacé dans seulement trois d'entre eux par un «Il» qui renvoie à Jésus. La singularité de cette incantation du nom est unique dans l'œuvre 
pascalienne. Dans Les Provinciales figurent 112 occurrences de "Jésus-Christ ", pas une seule de "Jésus ». Cette habitude se confirme dans les Pensées, où "Jésus-Christ » est employé 276 fois ; les deux seules mentions de « Jésus » seul, au fr. 419, n'apparaissent significativement que dans la formule "l'invocation du nom de Jésus». Un tel constat nous oriente vers une pratique liturgique, celle de la litanie, qui gouverne tout le «Mystère ». Dans un livre qu'il connaissait bien, les Heures de Port-Royal, Pascal récitait les «Litanies du saint nom de Jésus » : «Jesu potentissime / Jesu obedientissime / Jesu mitis et humilis corde / Jesu via et vita nostra.... » En revanche le fr. 751, qu'on a longtemps abusivement joint au "Mystère », est dépourvu de cette coloration liturgique, et se révèle tout autre.

Cette unité du poème se trouve en outre soutenue par la magie des dérivations (ou répétitions de mots de la même famille), ainsi que par la gravitation de mots illustrant un même thème: la solitude, le délaissement s'imposent ainsi dans tout le début du texte : " Jésus délaissé... Jésus seul en la terre... cet abandon dans l'horreur de la nuit... au milieu de ce délaissement universel... » Mais le lien le plus fort est constitué par le recours au polyptote, c'est-à-dire au jeu sur les flexions d'un même mot, ici sur celle du verbe "dormir »: "et ils dorment ", «les empêcher de dormir " (verset 2), "ses disciples dorment " (verset 7), «il ne faut pas dormir » (verset 8), «les trouvant dormant » (verset 9), « les trouvant encore dormant " (verset 10), " pendant que ses disciples dormaient " (verset 13), " pendant qu'ils dormaient» (verset 14), « voyant tous ses amis endormis » (verset 17). Une même musicalité donne à tout le poème une acoustique aisément reconnaissable, avec les lancinantes reprises des nasales, productrices d'ensorcèlement et de stupeur, et avec le règne de la voyelle aiguë qui caractérise le tragique, le "-i-», tout appropriée à ces moments d' "ennui», à ce " calice ». Le terme « agonie » assure l'ouverture et la clôture d'un poème dominé par le verbe «dormir » et par l'opposition "amis / ennemis ». Le verset 4 fait appel à deux rimes chères à la poésie religieuse baroque : « délices / supplices ", reliées par le passé simple « où il se perdit ». Qu'on écoute : s'adressant à « ses trois plus chers amis », « il les prie de soutenir un peu avec lui ». Ces perpétuelles reprises du «-i - »'entrelacent aux nasales: "Jésus sera en agonie jusqu'à la fin du monde. Il ne faut pas dormir pendant ce temps-là. » (verset 8). Ou encore : Jésus voyant tous ses amis endormis et ses ennemis vigilants... » (verset 17), ou la clausule du poème : "Jésus étant dans l'agonie, prions plus longtemps. » Une pareille maîtrise musicale se rencontre aussi au centre du célèbre fragment « Disproportion de l'homme » (fr. 230):

Nous brûlons du désir de trouver une assiette ferme, et une dernière base constante pour y édifier une tour qui s'élève à l'infini, mais tout notre fondement craque et la terre s'ouvre jusqu'aux abîmes [...] Notre raison est toujours déçue par l'inconstance des apparences, rien ne peut fixer le fini entre les deux infinis qui l'enferment et le fuient.

Cette stricte délimitation du « Mystère de Jésus » se trouve confirmée par la rigueur de la philologie : Pascal a écrit son poème sur le recto d'une grande feuille et l'a achevé tout en haut au verso de cette même feuille, dont tout le reste est demeuré vierge. Le fr. 751, que plusieurs éditeurs ont voulu lier au «Mystère " à cause de son ardent christocentrisme, a été rédigé en commençant une autre grande feuille : il constitue une "Pensée » indépendante, comme l'a jugé avec raison Louis Périer, qui - dans son recueil de "Pensées » inédites - le sépare du fr. 749 en insérant entre les deux une série de notations hostiles aux jésuites (fr. 750). 
17 L'incontestable indépendance des fr. 749 et 751 n'empêche pourtant pas ce dernier dans la manifeste variété de ses notations spirituelles - de contenir plusieurs retours sur l'Agonie. Le plus célèbre est la notation $4:$ "Je pensais à toi dans mon agonie, j'ai versé telles gouttes de sang pour toi. » Il s'agit de la sueur de sang, décrite par Luc (22, 44) comme constituée de "gouttes qui tombaient jusqu'à terre ». C'est donc dans l'Agonie plus encore qu'à la Croix que Pascal se sent aimé et s'espère prédestiné. Ce rôle prédominant de Gethsémani par rapport à la Passion, considérée comme distincte, s'exprime dès le verset $1 \mathrm{du}$ « Mystère » : "Jésus souffre dans sa Passion les tourments que lui font les hommes. Mais dans l'agonie ...» Des souffrances infligées par les hommes, qui n'abattent pas Jésus, se distingue infiniment l'effroyable douleur intérieure en face du péché du monde à laquelle Jésus s'est exposé volontairement. Même dans le cri "Lamma sabacthani » du Crucifié, Pascal ne voit que la reprise d'un psaume rempli de confiance en Dieu : «Ces paroles sont pleines d'espérance et non pas de désespoir.» (Abrégé..., n. 279). Quand les évangélistes peignent Jésus "si troublé, c'est quand il se trouble lui-même. Et quand les hommes le troublent, il est tout fort. » (fr. 347).

18 Cette mention de la sueur de sang se poursuit dans la huitième notation : "Veux-tu qu'il me coûte toujours du sang de mon humanité sans que tu donnes des larmes? » Le sens de l'adverbe «toujours » s'explique par le verset 8 du « Mystère » : "Jésus sera en agonie jusqu'à la fin du monde. Il ne faut pas dormir pendant ce temps-là. »C'est pourquoi le poème peut s'achever par «Jésus étant dans l'agonie et dans les plus grandes peines, prions plus longtemps. ». Il ne s'agit ici nullement du Corps mystique du Christ, de l'Église, qui traverse les souffrances au cours de l'histoire, unie à son Maître. Car il ne s'agit pas de souffrances prises en un sens général et indéterminé, mais d'un supplice clairement spécifié et qui n'eut ni n'aura jamais rien de comparable : l'Agonie de Jésus, strictement et uniquement personnelle. Aucun saint n'a fait face au Péché du monde, comme le Christ seul a pu le faire. Aucun saint n'a sué le sang sous le poids de l'intensité du supplice. Comment dès lors comprendre que cette Agonie durera jusqu'à la fin du monde? La réponse est fournie par la christologie oratorienne, telle qu'elle a été fixée par Bérulle et par l'un des maîtres spirituels de Pascal, Charles de Condren. Chacun des grands "états » ou "mystères » de la vie de Jésus surplombe l'écoulement du temps historique, parce qu'il demeure présent et actif dans l'âme du Christ: reviviscence traumatique constamment renouvelée et qui durera à jamais, à l'instar des cicatrices physiques de la Passion, «navrures d'éternité » (selon Bérulle), qui ne disparaîtront jamais. Pascal aurait donc pu écrire que «Jésus sera agonie perpétuellement ", mais sa perspective se limite au temps de l'Église militante, où les chrétiens sont appelés « à ne pas dormir » et à prier " plus longtemps ».

19 Cette interprétation de la formule «en agonie jusqu'à la fin du monde » se trouve confirmée - s'il en était besoin - par le dernier affleurement de l'Agonie dans les œuvres pascaliennes. Celui-ci se produit en novembre 1659 ou 1660 dans la Prière pour demander à Dieu le bon usage des maladies. Son douzième paragraphe s'ouvre sur la contemplation actuelle de Gethsémani : «Seigneur, ne permettez pas que je sois dans un tel éloignement de vous que je puisse considérer votre âme triste jusqu'à la mort, et votre corps abattu par le mort pour mes propres péchés, sans me réjouir de souffrir et dans mon corps et dans mon âme. Car qu'y a-t-il de plus honteux, et néanmoins de plus ordinaire dans les chrétiens et dans moi-même, qui, tandis que vous suez le sang pour l'expiation de nos offenses, nous vivons dans les délices. " C'est bien aujourd'hui, je le 
souligne, pendant la vie de Pascal, pendant que celui-ci le trahit, que le Christ traverse un supplice à nul autre pareil, qui lui fait "suer le sang ", supplice infligé librement par « une main toute-puissante » et qu' «il faut être tout-puissant pour soutenir », pour supporter, pour y résister (c'est l'ouverture même du « Mystère de Jésus »). Un peu plus loin, la Prière reprend un énoncé $\mathrm{du}$ " Mystère » : «Vous vous êtes exposé à la colère de Dieu », qui renvoie au «Jésus était délaissé seul à la colère de Dieu » (verset 4).

C'est à cette Agonie actuelle du Christ que le chrétien peut demander à être associé, sans toutefois que sa douleur intérieure pour ses péchés et pour ceux du monde puisse être réellement comparable à l'Agonie de Jésus. De là la fervente supplication qui clôt la Prière:

Entrez dans mon cœur et dans mon âme, pour y souffrir mes souffrances, et pour continuer d'endurer en moi ce qui vous reste à souffrir de votre Passion, que vous achevez dans vos membres jusqu'à la consommation parfaite de votre Corps; afin qu'étant plein de vous, ce ne soit plus moi qui vive et qui souffre, mais que ce soit vous qui viviez et qui souffriez en moi, ô mon Sauveur; et qu'ainsi, ayant quelque part à vos souffrances, vous me remplissiez entièrement de votre gloire, dans laquelle vous vivez avec le Père et le Saint-Esprit, par tous les siècles des siècles.

Il s'agit là de la reprise d'un verset célèbre de saint Paul: "Ce qui manque aux souffrances du Christ, je l'achève en ma chair. (Colossiens 1, 24). Cette reprise reste ici très générale, mais elle est spécifiée dans deux autres passages de la Prière, où l'orant supplie le Christ de plonger son âme "dans la tristesse " pour ses propres offenses (\$10). Cette tristesse est bien celle de l'Agonie : «Mettez en moi une tristesse conforme à la vôtre.» (\$13). C'est cette communion dans la tristesse pour les péchés qui rend compte d'une notation hardie du fr. 751 : «Loin de m'abhorrer, il se trouve honoré que j'aille à lui et le secoure.» (notation 19), contrairement aux trois apôtres endormis au Jardin des oliviers.

On le voit, aucun autre "mystère » de la vie de Jésus n'a suscité chez Pascal un ensemble de textes qui approche des affleurements de l'Agonie. Le jeune théologien est visiblement habité par Gethsémani. Il nous faut maintenant nous interroger sur les lectures dont a été nourrie cette floraison, avant de passer à l'approfondissement de la théologie qui l'inspire.

\section{Les origines de la méditation pascalienne}

Les textes sur Gethsémani sont évidemment d'abord nourris des versets de la Bible : des évangiles et du prophète le plus cher à Pascal, Isaïe ${ }^{8}$. Comme cela a été rappelé, il faut leur ajouter les deux ouvrages de jansénius : le Tetrateuchus, ou commentaire des quatre évangiles, et la Series vitae Jesu Christi, ainsi que l'Historia et concordia evangelica d'Antoine Arnauld.

Une minuscule énigme demeure : d'où provient la citation "Turbare semetipsum ». A-telle été forgée par Pascal, qui aurait fondu deux versets de l'évangile selon saint Jean ? Lorsqu'il raconte la mort de Lazare et l'affliction du Christ devant le cadavre de son ami, l'évangéliste écrit : «il se troubla » $(11,33)$, et un peu plus loin : «à nouveau Jésus frémissant en lui-même ». Ce que la Vulgate a traduit : «turbavit se ipsum » et "rursus fremens in semetipso ». En fait l'augustinien qu'est Pascal fait simplement passer à l'infinitif pour la commenter une citation de saint Augustin. C'est celui-ci qui est à l'origine du premier verset du «Mystère ». Dans son traité 49 sur l'évangile de 
saint Jean (n. 18-19), l'évêque d'Hippone explique le sens de ce trouble du Christ: " fremuit spiritu et turbavit semetipsum»:

J'ignore ce qu'il a voulu nous faire entendre. Qui en effet pourrait le troubler, si ce n'est lui-même ? [...] Toi, tu es troublé sans le vouloir; le Christ, lui, a été troublé parce qu'il l'a voulu [...] Le Christ a été saisi de tristesse, cela est vrai, mais parce qu'il l'a voulu : en son pouvoir était d'être affecté de telle manière ou de ne pas être affecté [...]

Que veut dire que le Christ se troubla lui-même, si ce n'est pour te faire comprendre que toi, tu dois être troublé puisque tu es accablé et écrasé d'une lourde masse de péché ${ }^{\text {? }}$

En homme d'un temps imprégné de stoïcisme, Augustin considère qu'un homme parfait exerce la maîtrise de sa volonté sur ses passions ou sur ses souffrances. Par conséquent l'homme-Dieu ne peut être affecté que par « les tourments qu'il se donne à lui-même ", volontairement. Pascal retrouvait aussi le "parce qu'il l'a voulu» (quia voluit) chez ce connaisseur exceptionnel d'Augustin qu'était Jansénius. Celui-ci commente le verset "Il commença à être saisi de tristesse " (Matthieu 26, 37) et écrit: "parce qu'il a été saisi de tristesse non pas brusquement et contre sa volonté, comme cela nous arrive, mais de son plein gré, quand, comment et combien il le voulut ${ }^{10}$. " C'est l'évêque d'Ypres qui applique au trouble de l'Agonie le développement d'Augustin sur la tristesse du Christ lors de la mort de Lazare. On pourrait traduire sa pensée en affirmant que Jésus a entrepris de s'exposer à la souffrance choisie par lui-même. Nous sommes ici au jaillissement originel du début du « Mystère de Jésus » :

Jésus souffre dans sa Passion les tourments que lui font les hommes. Mais dans l'agonie il souffre les tourments qu'il se donne à lui-même. Turbare semetipsum. C'est une souffrance d'une main non humaine, mais toute-puissante. Et il faut être toutpuissant pour le soutenir.

Ainsi s'affirme avec éclat la singularité de l'Agonie, fermement distinguée - on l'a vu de la Passion, qui la suit. L'intensité du supplice précise le «quantum voluit» du Tetrateuchus, en faisant appel à la pensée de Condren : «Je crois qu'il a plus souffert en ce mystère qu'en les autres endroits de sa Passion. »

La netteté et le caractère attendu de ces filiations rendent bien pu probable que Pascal se soit inspiré du dominicain Louis Chardon, auteur de La Croix de Jésus (1647) et de Méditations sur la Passion de Notre Seigneur Jésus-Christ pour tous les jours de l'année (1650), contrairement à ce qu'a proposé le père Jean-Christophe de Nadaï dans son Jésus selon Pascal. Le dominicain revient sans cesse à la théologie de son maitre, saint Thomas, qui limite la souffrance du Christ à «la partie sensitive» de son âme et qui n'est jamais évoqué par Pascal à propos de Gethsémani. Les Méditations sont tout autres que les textes pascaliens : elles abusent des qualificatifs du paroxysme; la "Méditation 73 " montre « Jésus baigné dans son sang durant sa sueur » (c'est son titre) ; l'auteur s'attendrit comme un médiéval sur "le pauvre Jésus », «l'aimable Jésus ». Enfin que de redites! Pas moins de huit «Méditations » consacrées à la sueur de sang, qui n'est même pas mentionnée dans le " Mystère ${ }^{11}$ !

De même, on ne retrouve chez Pascal ni le lexique, ni les thèmes de La Croix de Jésus, par exemple le leitmotiv de "l'inclination à la Croix », central dans le premier entretien. Le chapitre 22 de celui-ci a pour titre "L'amour séparant Jésus du Saint-Esprit et de soimême ", mais aucune des formules hardies de ce chapitre ne passe chez Pascal. Le père de Nadaï en cite que Jésus est "à soi-même cause d'abandonnement » et qu' « il veut être à soi-même principe de tourment et instrument de martyre ». Mais nous venons de 
voir que cette thématique augustinienne était répandue. Le Jésus selon Pascal propose du "Mémorial» (fr. 742) une lecture paradoxale où la partie centrale serait constituée de paroles prononcées par Jésus, séparé de son Père lors de l'Agonie. Mais cette interprétation est contredite par maints aspects du poème: la datation solennelle et l'annonce des traces d'une expérience personnelle intense de la présence divine. Un verset comme "Joie, joie, joie, pleurs de joie» serait attribué au Christ, ce qui constituerait un hapax chez Pascal, et une nouveauté bizarre absente de tous ses autres textes sur l'Agonie, alors qu'il est on ne peut plus naturel chez le bénéficiaire d'une grâce exceptionnelle (on retrouve ces mêmes "pleurs de joie » chez saint Siméon le nouveau théologien lors d'une expérience étonnamment similaire). Enfin il faut souligner l'absence totale de marques de changement d'énonciateur, de Pascal à Jésus. Au contraire les deux citations de la prière sacerdotale du Christ (Jean 17, 25 et 17, 3) s'expliquent on ne peut mieux par l'application que le « converti » s'en fait.

Ainsi deux conclusions semblent s'imposer : aucun indice probant d'une influence de Chardon n'est décelable dans les œuvres pascaliennes; en second lieu, la lecture peu naturelle du « Mémorial » qui s'autorisait du dominicain, n'est plus soutenable. Dès lors les traces écrites de la « Nuit de feu» du 23 novembre 1654 ne viennent pas s'ajouter aux textes gravitant autour de l'Agonie.

30 L'hypothèse d'une influence de Chardon pose la question de la lecture par Pascal d'autres ouvrages de spiritualité de son époque. Question légitime, mais qui appellerait de longues enquêtes, avec des résultats pas forcément sûrs et de grand intérêt. Ainsi le père Pietro Stella a décelé dans « Le Mystère de Jésus » quelques correspondances avec le Manuel de méditations (1650) du jésuite Giovanni Busaeus. Ce dernier écrit: "Il cherche quelque consolation et soulagement de ses disciples, et ils dorment [...] Jésus prie [...] c'est dans les plus grandes afflictions qu'il faut prier.» Nous voici proches des versets 2 et $20 \mathrm{du}$ poème pascalien. Néanmoins, en connaisseur de la surabondance de la littérature méditative des XVI ${ }^{e}$ et XVII ${ }^{e}$ siècles, le père Stella doute lui-même de ce qu'on peut conclure de ces rapprochements, tant il peut s'agir de lieux communs, de formules répandues dans toute une époque. Va dans le sens de ce scepticisme la troisième coïncidence qu'il propose: "Hélas! Quel est ce jardin [...] on trouve des délices dans les autres, mais en celui-ci il n'y a que tristesse et effroi. » Le verset $4 \mathrm{du}$ " Mystère oppose, lui, « délices » et « supplices », où quiconque est familier de la poésie baroque, hantée par l'opposition ou la coïncidence des contraires, reconnaît un de ces jeux de rimes qui y sont fréquents : « délices / supplices », comme « miel / fiel ». Frère d'une jeune poétesse prodige, Pascal a en mémoire ces jeux verbaux récurrents. Enfin, dans son préambule, Busaeus vantait la pratique ignacienne de la «composition de lieu » pour soutenir la méditation, avec appel à l'imagination. Au contraire, le poème pascalien, loin de recourir à l'imagination, se tient au plus près des récits bibliques et s'attache à ce que son biographe, Amelote, disait de Condren: "L'occupation continuelle de son âme était l'intérieur du Fils de Dieu.» Avec le "Mystère » nous sommes en présence d'une « adhérence » condrénienne : le méditant-orant s'identifie à l'angoisse et à la prière de Jésus ${ }^{12}$, à son âme, et non au cadre extérieur et à des détails inventés.

31 Le père Stella doutait finalement que Pascal ait lu l'ouvrage du jésuite. Jean Mesnard ne s'exposait à aucun doute semblable, en démontrant que l'auteur des Pensées connaissait fort bien le Discours en forme de Lettre de Notre Seigneur Jésus-Christ à l'âme dévote du chartreux Jean Lansperge (1489-1539). La traduction de ce Discours, publiée en 
février 1657, émanait de Port-Royal et était publiée par son éditeur attitré, Savreux. Presque tout de suite, Jacqueline Pascal, dans son Règlement pour les enfants, l'inscrivait sur la liste des livres à faire lire aux petites filles dont elle avait la charge dans le monastère. Parmi les diverses correspondances que Jean Mesnard met en lumière avec les textes de Pascal - si le « Mystère de Jésus » n'est pas concerné - le fr. 751 en contient plusieurs. Le seul de ces rapprochements qui ait trait à l'Agonie est la notation $4:$ : Je pensais à toi dans mon agonie, j'ai versé telles gouttes de sang pour toi. » Le chartreux avait écrit : « avant même que vous fussiez au monde, j'avais de l'amour pour vous et je souffrais pour vous des tourments sans nombre [...] Je voulais verser pour vous tout mon sang; ce que j'ai effectué si ponctuellement qu'il ne m'en est pas resté une goutte. » Certes Lansperge vise ici l'ensemble de la Passion, et non à la seule Agonie, mais l'idée d'une présence personnelle du pécheur à la conscience du Christ souffrant est bien présente. L'ensemble des correspondances mises en lumière fait preuve : Pascal s'est bien nourri du chartreux ${ }^{13}$. Mais c'est lui seul qui introduit l'insistance sur l'Agonie.

Bref, la méditation pascalienne sur Gethsémani s'est développée en s'appuyant sur un essaim de textes chers à Port-Royal: la Bible, les Heures de Port-Royal, Augustin, Jansénius, Condren, Arnauld et un représentant d'un Ordre révéré par les spirituels du monastère : la Chartreuse ${ }^{14}$.

\section{Essai de théologie pascalienne de l'Agonie}

Jeudi saint ${ }^{15}$ ». Elle fait suite à l'institution du mystère eucharistique, si central à PortRoyal du Saint-Sacrement et qui a inspiré la magnifique lettre à Charlotte de Roannez de la fin octobre 1656 sur les voiles qui couvrent Dieu. De même, les paroles testamentaires qu'on a nommées la "Prière sacerdotale » du Christ (Jean, 13-17) se trouvent bien présentes à l'esprit de Pascal : qu'on en juge par le « Mémorial (fr. 742)! En tout cela il s'agit d'une courte séquence temporelle - cinq à six heures - et du petit massif de textes évangéliques qui ont le plus vivement parlé à l'auteur du « Mystère de Jésus ». Quelle est donc la théologie qui anime le dernier de ces épisodes, Gethsémani ? L'union en Jésus-Christ de la divinité et de l'humanité est considérée par Thomas 
d'Aquin comme le plus inattendu et le plus incompréhensible des mystères qui constituent la foi chrétienne ${ }^{16}$. Une fois que l'enquête historique et la grâce divine ont fait percevoir dans la personnalité déroutante de Jésus des signes indubitables de son origine transcendante, rien ne devient plus raisonnable que de se mettre passionnément à son écoute. Comment procède alors le théologien, et même le chrétien, qui, dès qu'il réfléchit sur le contenu de sa foi, se constitue en théologien en herbe. Il ne peut que scruter tous les textes bibliques qui parlent de la personnalité du Christ, de ses paroles et de ses actions, pour tenter d'en proposer la synthèse la plus rigoureuse possible. En cela il ne fait que suivre l'exemple des premières générations chrétiennes qui, peu à peu, face aux approximations ou aux aberrations qui surgissaient, ont défini dans les Conciles œcuméniques les limites à l'intérieur desquelles l'ensemble de l'Église reconnaissait que sa foi se situait. Pour mieux cerner la théologie de Pascal, arrêtons-nous un instant sur la plus célèbre de ces synthèses, celle de saint Thomas d'Aquin, la plus répandue dans l'Église catholique : l'auteur du « Mystère de Jésus » est-il thomiste?

\section{Lâme du Christ selon saint Thomas}

37 En s'appuyant sur divers passages des évangiles synoptiques et surtout sur l'évangile de saint Jean, Thomas d'Aquin conclut que l'âme du Christ, unie au Verbe divin, a toujours possédé la vision béatifique (elle voit Dieu), la "science infuse ", c'est-à-dire une connaissance directe de tous les intelligibles (sans avoir à les extraire comme nous des données sensibles). Malgré ces privilèges, elle a progressé dans une science acquise, comme nous, car le Verbe divin a assumé une nature humaine intégrale, avec toutes ses activités propres ${ }^{17}$. De là le verset de Luc : enfant, "Jésus progressait en sagesse » (2, 52).

38 Certes l'âme du Sauveur, humaine, reste incapable de " comprendre » l'essence divine, qu'elle « voit » (q.10, a.1, c.). Mais la nature divine du Christ, elle, comprend l'infinité de cette essence (q.10, a.1, 3m). Dans le Verbe, l'âme de Jésus voit tout ce qui était, est et sera fait, dit ou pensé chez les créatures (q.10, a.2).

39 Toute l'âme du Christ a-t-elle souffert dans sa Passion (q.41, a.7) ? Même au cœur de ses souffrances, elle n'a cessé d'éprouver la joie béatifique (q.47, a.8). Mais lorsque le Christ était voyageur sur cette terre, il n'y avait pas de rejaillissement de sa gloire de la partie supérieure de son âme sur sa partie inférieure, ni de l'âme sur le corps (q.46, a.8). Donc l'âme pâtissait uniquement selon ses puissances inférieures (q.46, a.7).

40 Une telle élaboration théologique - si elle rend compte de nombreux textes évangéliques - s'expose à trois grandes objections. D'une part elle est tributaire de la conception aristotélicienne de l'âme, avec ses trois étages (intellective, sensitive et végétative); les souffrances de la Passion ne dépasseraient pas l'étage sensitif, tandis que l'étage intellectif demeurerait irradié de la joie béatifique. Un augustinien comme Pascal, convaincu de l'unité indivisible de l'âme, pouvait-il adopter pareille théorie ? Il lisait aussi, dans Les Passions de l'âme de Descartes, qu'« il n'y a en nous qu'une seule âme, et [que] cette âme n'a en soi aucune diversité de parties. » (I, 47).

41 En second lieu, cette coexistence d'une joie infinie et des souffrances de la Passion ne relève-t-elle pas d'une forme sophistiquée de docétisme, d'une mascarade ${ }^{18}$ ?

Enfin, l'accablante tristesse de Jésus exposé seul au péché du monde peut-elle se situer dans la partie sensitive de l'âme? Cette noble tristesse de l'amour déçu et bafoué ne 
peut qu'affecter la cime même de l'âme. Il semble bien que Thomas d'Aquin ait perçu la difficulté : d'abord parce qu'il évite de consacrer une question de la Somme à l'Agonie, qui mettait à rude épreuve sa construction, alors qu'il en compose une sur l'épisode qui forme diptyque avec elle, la Transfiguration (q.45). Lors de la Transfiguration, Jésus n'a pris avec lui que les trois mêmes disciples que lors de l'Agonie, et cette théophanie doit prémunir les trois apôtres contre l'ébranlement causé par les souffrances de l'Agonie. En second lieu, parce que, lorsqu'il est forcé de s'interroger sur l'existence d'une tristesse de Jésus, il ne s'en tire que par des contorsions qui ne rendent nullement compte du paroxysme des récits de Gethsémani. Selon lui, au Mont des oliviers, l'âme du Christ, irradiée par le Verbe divin, ne pouvait que s'opposer victorieusement aux souffrances. Aussi s'appesantit-il sur le verset «Il commença à s'attrister ». Jésus alors n'aurait connu que ce que Thomas appelle une "pro-passion ", c'est-à-dire une passion qui, commencée dans l'appétit sensible, ne pouvait s'étendre au-dessus (q.45, a.4, c. et $1 \mathrm{~m})$. Le caractère effrayant du "triste jusqu'à la mort » et de la sueur de sang n'est-il pas purement et simplement éludé ?

\section{Pascal contre le risque de docétisme}

Comme Thomas, l'auteur du « Mystère de Jésus » a perçu que le régime général d'union des deux natures dans la personne du Christ tient compte de la mission de salut qui lui est impartie. C'est pour rendre possible son cheminement en inconnu parmi les hommes que la gloire du Verbe, qui naturellement irradiait son âme, n'a pas rejailli sur le corps. Néanmoins ce régime habituel de retenue a été fugitivement transgressé lorsque cela était nécessaire à tel dessin particulier. Ainsi en fut-il - selon Thomas (q.45, a.2, c.) - lors de la naissance miraculeuse du Christ, lors de sa marche sur les flots du lac de Tibériade (Matthieu 14, 25), lorsqu'il échappa à ceux qui voulaient le précipiter du haut d'un escarpement (Luc, 4, 29-30) et dans la théophanie de la Transfiguration.

Le texte du "Mystère de Jésus » invite à retrouver dans l'Agonie cette souplesse fugace du régime général de l'union. Certes l'intelligence de l'âme christique demeurait irradiée par le Verbe, et se savait ainsi sauvant le monde (versets 3 et 45). C'est pourquoi Jésus peut dire dans le fr. 751 : «Je pensais à toi dans mon agonie, j'ai versé telles gouttes de sang pour toi.» (notation 4). Mais Pascal prend au sérieux l'abîme effrayant des souffrances, si effroyable qu' « il faut être tout-puissant pour le soutenir (fr. 749, verset 1). Car Jésus-Christ, juste, a fait face, non au péché, mais aux conséquences de tout le péché du monde, c'est-à-dire à un entier éloignement de Dieu, à une mortelle solitude : «Il a été fait péché pour moi : tous vos fléaux sont tombés sur lui » (fr. 751, notation 19).

Pour éviter tout risque de docétisme, le "Mystère " et l'Abrégé posent que, dans l'Agonie, l'affectivité spirituelle de la nature humaine se trouva dans « la destitution de toute consolation et divine et humaine» (Abrégé, 215). Au Mont des oliviers, "Jésus était délaissé seul à la colère de Dieu » et «Il souffre cette peine et cet abandon dans l'horreur de la nuit» (fr. 749, versets 2 et 5). Cette totale absence de Dieu dans l'affectivité supérieure permet seule de saisir la "douleur excessive " et la plainte inouïe : "Mon âme est triste jusqu'à la mort. » (verset 6). C'est cet affolement dans la souffrance qui explique la seule plainte personnelle que Jésus ait proférée dans la totalité des évangiles (verset 6) et la recherche répétée d'un consolation humaine, recherche qui constitue le leitmotiv du « Mystère » (versets 2, 3, 5, 6, 7, 9, 11, 12, 17). 
On aboutit à cette confondante anomalie : "Jésus a prié les hommes et n'en a pas été exaucé » (verset 12). Pour rendre plus palpable cette absolue déréliction, le "Mystère " passe sous silence la consolation fugace de l'Ange, dont pourtant l'Abrégé faisait état (215). Du même coup, la sueur de sang n'est pas mentionnée.

\section{«Abandonné ... La face en terre »}

L'Agonie illustre la conception catastrophique que Pascal, comme tout Port-Royal, se fait de l'Incarnation. Loin d'insister, comme les Grecs, sur la divinisation de la nature humaine, il y voir l'avilissement du Verbe. D'autre part, la christologie pascalienne est foncièrement dynamique : la Sagesse éternelle est descendue de sa gloire trinitaire en assumant une véritable nature humaine, elle est venue vivre " humiliée " parmi nous. Le moment le plus bas de cette trajectoire, c'est non pas la Croix, mais l'Agonie, où

211. Il prie.

212. La face en terre.

213. Trois fois [...]

215. [...] Et dans cette agonie, il sue le sang.

L'Abrégé, pour la formule « La face en terre », fond Matthieu 26, 39 («il tomba sur sa face ») et Luc 22, 44 « sa sueur devint comme des gouttes de sang tombant à terre »). Les « Trois fois » sont communes aux trois évangiles synoptiques, chiffre à la fois réel et de portée symbolique qui solennise ce moment unique de l'humiliation paroxystique du Verbe incarné. Il va mourir «trahi par un des siens, renié par l'autre et abandonné par tous » (fr. 736).

Dans le « Mystère de Jésus », au polyptote du verbe « dormir » s'en tresse un autre sur l'abandon et le délaissement : « ils le laissent », « délaissé seul » (verset 2), « au milieu du délaissement universel » (verset 9), renforcé par «Jésus est seul en la terre [...] Le ciel et lui sont seuls dans cette connaissance (verset 3 ) et « Il souffre seul cette peine et cet abandon dans l'horreur de la nuit » (verset 5).

Voudra-t-on réduire le privilège de l'Agonie en rappelant qu'à la Croix aussi le Christ a été abandonné, "sans consolation ». Mais Pascal fait partie de ceux qui interprètent le Lamma sabactani en le lisant comme le premier verset d'un psaume d'espérance, le Psaume 22. Aussi écrit-il dans l'Abrégé :

279. «Éli, Éli, lamma sabactani ?» c'est-à-dire : "Mon Dieu, mon Dieu, pourquoi m'avez-vous délaissé ?»- savoir en sa nature humaine, abandonné à tous les tourments des bourreaux, et de ses ennemis, sans consolation. Et il s'adresse à Dieu pour demander la cause de cet abandon [...] Et même ce discours peut être entendu comme une prière que Jésus fait au Père de se souvenir de la fin pour laquelle il l'afflige et l'abandonne, comme disant: «Mon Dieu, pourquoi m'avez-vous délaissé ? vous savez, mon Dieu, que c'est pour le salut du monde, appliquez donc le fruit de ce sacrifice au genre humain, auquel vous l'avez destiné. » Et ces paroles sont pleines d'espérance et non pas de désespoir, car il dit: "Mon Dieu, mon Dieu ! » or, Dieu n'est point le Dieu des morts, ni des désespérés ${ }^{19}$ ?

Dans toute sa Passion, que Pascal - nous y avons insisté - distingue de l'Agonie, la nature humaine du Christ résiste avec constance aux tourments que lui infligent les bourreaux, tourments venus de l'extérieur. Mais à Gethsémani son âme est dévastée par un supplice tout intérieur, l'affrontement au péché du monde, si paroxystique qu'elle est submergée un moment par la détresse la plus indicible, ce qui provoque la 
sueur de sang, remplacée dans le « Mystère » par une effrayante désolation intérieure : " Jésus dans l'ennui » et « Mon âme est triste jusqu'à la mort ».

La formule "sans consolation" implique-t-elle aussi le retrait de la grâce divine accordée aux hommes justes? La question se pose, parce que Pascal parle habituellement de la "consolation" apportée par la grâce, en particulier dans la "Lettre sur la mort", et dans la Prière pour demander à Dieu le bon usage des maladies, $\S 11)$. Il semble que non, car Jésus est toujours demeuré un juste. Il faut donc restreindre ici le sens à la consolation issue de l'irradiation du Verbe dans l'âme. Au Jardin des oliviers, Jésus était évidemment toujours un juste, mais il a vécu une "nuit » dont nous avons vu qu'elle avait été comparée à celle dont parle saint Jean de la Croix à propos de la vie mystique.

Sur la Croix, contrairement à Gethsémani, Jésus a reçu quelque consolation humaine de la présence de sa mère, de l'apôtre Jean et d'un petit groupe de femmes fidèles (Jean 19, 25-27).

Dans l'Agonie, les leitmotive de la solitude et de l'abandon nous installent dans l'univers du péché, c'est-à-dire dans «l'horreur de la nuit " (verset 5), la nuit et la solitude que le Christ lui-même a présentées comme des images de l'enfer, de la totale absence de Dieu. Rien d'étonnant, dès lors, que l'on retrouve Gethsémani en filigrane dans toute description de l'errance humaine loin de Dieu : «En voyant l'aveuglement et la misère de l'homme, en regardant tout l'univers muet et l'homme sans lumière abandonné à lui-même et comme égaré dans ce recoin de l'univers [...] j'entre en effroi... » (fr. 229). Les pécheurs eux aussi sont « laissés » (fr. 265, 644), « abandonnés » (fr. 168). Au Jardin des oliviers, Jésus s'est volontairement immergé dans l'effroi du péché.

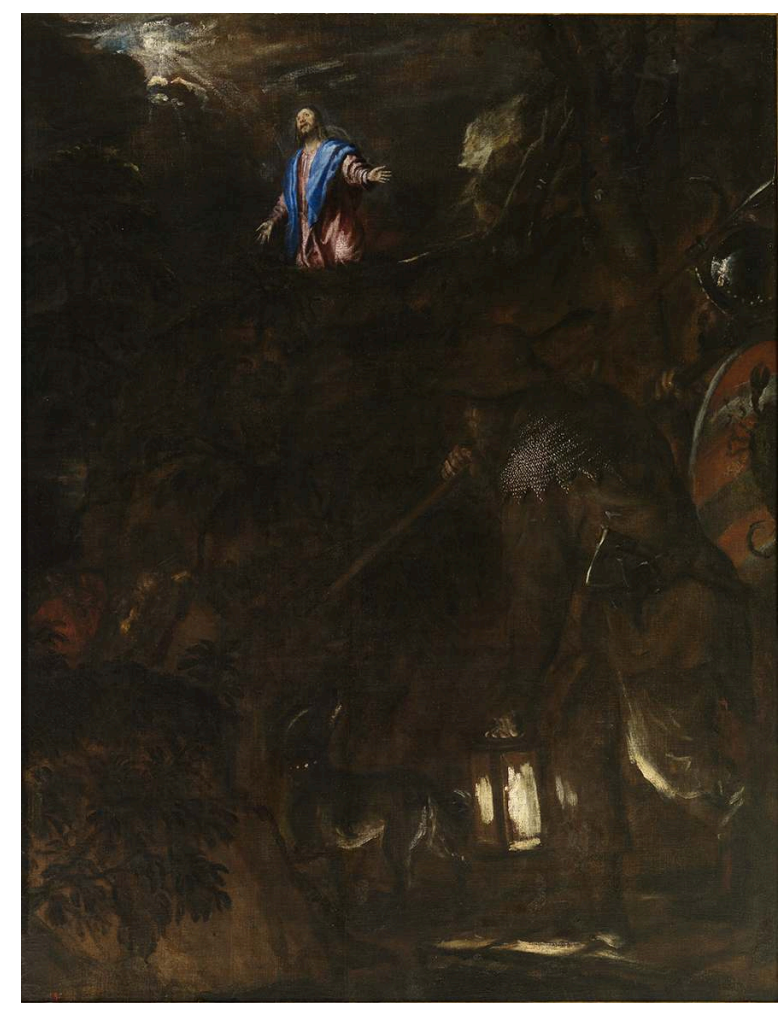

Le Titien, Agonie dans le jardin (Musée du Prado). 


\section{Les « appropriations divines »}

54 réserver à l'une des Personnes divines des attributs ou des opérations communs aux trois et qui caractérisent en réalité l'unicité divine. Cette pratique, appelée par les théologiens "appropriation", fait qu'on a traditionnellement approprié au Père la Puissance, au Fils la Sagesse et au Saint-Esprit l'Amour, bien que la Puissance, la Sagesse et l'Amour soient communs à toute la Trinité. Pourquoi le recours à ce procédé qui, au lieu de souligner l'unicité divine, pourrait égarer dans un trithéisme? C'est à cause de notre faiblesse devant l'incompréhensible mystère du Dieu unique et trinitaire: si, à partir du créé, nos intelligences peuvent s'élever à l'essence divine et à ses attributs (unité, infinité, éternité, etc.), comme l'illustrent par exemple la Somme contre les Gentils de saint Thomas d'Aquin et les Méditations métaphysiques de Descartes, elles ont du mal à entrevoir la distinction des Personnes. Celle-ci n'est fondée que sur la diversité des relations d'origine de ces Personnes. C'est pour rendre quelque peu perceptible ce secret qu'on a attribué à chaque Personne des attributs qui ont quelque rapport à sa relation d'origine. Ainsi au Père, origine des deux autres Personnes convient l'appropriation de la Toute-Puissance; au Fils engendré éternellement et intellectuellement comme conception de la pensée du Père, conviennent la Sagesse et les qualités intellectuelles, comme la Vérité) ; à l'Esprit, lien d'amour du Père et du Fils, ce qui relève de la volonté, voilà pourquoi Jésus lui-même le désigne comme le «Consolateur » (Jean 14, 16), ce qui pourtant lui est commun avec le Christ (1 Jean 2, 1). De là la définition de saint Thomas : "Manifester les Personnes au moyen des Attributs de l'essence [du Dieu unique], c'est ce qu'on nomme appropriation.» (Somme de théologie, Ia pars, q.39, a.7, c.).

Quand Pascal écrit, dans le "Mystère de Jésus », que "Jésus était délaissé seul à la colère de Dieu ", cela ne signifie nullement qu'il y a le moindre désaccord entre un Père qui exigerait une compensation aux péchés du monde et un Fils qui serait sa victime. En réalité le dessin miséricordieux d'arracher l'humanité à l'absence de Dieu et de lui apporter le salut est commun aux trois Personnes du Dieu unique. «Jésus ne regarde pas dans Judas son inimitié, mais l'ordre de Dieu qu'il aime » (verset 18). Néanmoins une appropriation traditionnelle donne au Père la Création du monde, au Fils sa Recréation et à l'Esprit sa Glorification. C'est pourquoi le premier verset du poème attribue le fait de "se troubler lui-même" non seulement à la volonté humaine de Jésus, mais aussi à sa Personne divine: "C'est un supplice d'une main non-humaine, mais toute-puissante. Et il faut être tout-puissant pour le soutenir.» Ainsi la seconde Personne de la Trinité semble le seul véritable acteur de la Rédemption, et cela non seulement parce que c'est sa nature humaine qui souffre "pour la multitude en rémission des péchés » (Matthieu 26, 28), mais par appropriation. En réalité, c'est toute la Trinité qui destitue l'âme du Christ de toute consolation divine.

\section{«Dans l'incertitude de la volonté du Père »}

Ce verset du «Mystère de Jésus» (11) laisserait pour le moins perplexe un théologien thomiste. Dans la vision pascalienne de l'Agonie, il suppose que l'âme du Christ - déjà destituée de toute consolation de la volonté - a vu, un court moment, son intelligence obnubilée enveloppée dans un nuage d'obscurité. De là un instant de désarroi, au paroxysme de la souffrance, et l'étonnante prière: «Père, si tu veux, emporte cette 
coupe loin de moi » (Luc 22, 42), ou « Père, tout t'est possible, emporte cette coupe loin de moi »" (Marc 14, 36). Le "Mystère » en conclut logiquement "Jésus prie dans l'incertitude de la volonté du Père, et craint la mort. Mais l'ayant connue, il va audevant s'offrir à elle. Eamus [Matthieu 26, 36]. Processit [Jean 18, 4]. » Pascal atténue ce verset un peu plus loin: "Il ne prie qu'une fois que ce calice passe, et encore avec soumission, et deux fois qu'il vienne, s'il le faut. »

Le théologien thomiste envisagera un éclair de révolte de la nature humaine, dans sa partie sensible, contre la mort, mais dont le Christ triomphe, vu la connaissance qu'il ne cesse de partager avec le Père du dessein de salut sur le monde. Saint Thomas écrit : "Il n'y a pas eu d'agonie chez le Christ quant à la partie rationnelle de son âme ${ }^{20}$.» Cette raideur s'explique par le souvenir de l'hérésie monothélite, au VII siècle : Thomas cite en effet le concile œcuménique de Constantinople, en 680-681, qui a condamné le monothélisme (des deux mots grecs monè et thelesis, qui signifient «une seule volonté »). Ce dernier réagissait précisément à la prière de Jésus au Jardin des oliviers en affirmant qu'il n'y avait en lui qu'une seule volonté, celle du Verbe. En effet, on ne pouvait imaginer dans l'âme christique une volonté humaine qui s'opposerait à Dieu, puisque la nature humaine est divinisée par l'Incarnation. La réponse au monothélisme vint de saint Maxime le confesseur (vers 580-662) : la révolte instinctive de la nature humaine, avec la prière « Si cette coupe ... » se situe non au niveau de la volonté libre, mais au plan infra-moral d'un mouvement naturel de la " chair qui craint la mort ». On entre au contraire dans l'espace moral des volontés libres et des décisions délibérées avec la fin du verset controversé : « Cependant, Père, que se fasse non ma volonté, mais la tienne. » (Luc 22, 42). Saint Thomas reprendra à peu près la même explication, en la situant dans une psychologie aristotélicienne (q.18, a.6).

Pour Pascal comme pour Maxime, ce moment de trouble marquait la réalité de l'Incarnation et appelait au courage et à la marche en avant ${ }^{21}$. Mais l'auteur du «Mystère de Jésus » n'était nullement monothélite: comme l'atteste le «Lettre sur la mort de son père ", il ne nie pas l'existence d'une volonté humaine dans l'âme du Christ, mais il la voit comme obnubilée brièvement par le paroxysme inouï de la souffrance. On retrouve là sa conviction que le salut du genre humain s'est opéré à Gethsémani beaucoup plus qu'à la Croix, dans ce jardin « où il s'est sauvé et tout le genre humain » (verset 4 ) et où « pendant que ses disciples dormaient, [il] a opéré leur salut. » (verset 13).

Cette affirmation que le Christ s'est sauvé lui-même paraît s'opposer à la synthèse de saint Thomas, selon laquelle Jésus a sauvé l'humanité, mais pas lui-même, comme «le soleil éclaire, et n'est pas éclairé » (q.22, a.4). Mais le docteur angélique nuance son affirmation dans la réponse à la seconde objection : «Le Christ a obtenu par sa Passion la gloire de sa Résurrection, non en vertu du sacrifice, offert par mode de satisfaction, mais en vertu de la dévotion qui lui a fait supporter sa Passion par charité.» Dans le "Mystère ", "il s'est sauvé " renvoie à l'entrée dans la gloire, selon la pensée déjà énoncée en 1651 dans la "Lettre sur la mort ", qui cite Luc 14, 26: «Il a fallu qu'il ait passé par les souffrances pour entrer en sa gloire. Et, quoiqu'il fût Fils de Dieu, il a fallu qu'il ait appris l'obéissance. » Cette citation de l'évangile de Luc est immédiatement suivie - nous l'avons vu - de l'allusion à Gethsémani : « Mais au jour de sa chair, ayant crié avec un grand cri, il a été exaucé pour sa révérence ; et Dieu l'a ressuscité et envoyé en gloire [...] C'est ce que Jésus-Christ a obtenu.» La «révérence» de la «Lettre » pascalienne est toute proche de la « devotio » de la Somme de théologie. 


\section{Une théologie datée : la « satisfaction vicaire »}

la théologie des Pères grecs, qui insistent sur la divinisation de l'humanité pécheresse $\mathrm{du}$ seul fait de l'Incarnation: par la seule présence du Verbe, la nature humaine ramassée dans le Christ, est divinisée, et donc sauvée. Nous sommes en présence d'une théologie de l'identification, et non de la substitution. Pascal, lui, reprend les catégories de la théologie latine de son temps, devenue classique depuis saint Anselme (1033-1109) et saint Thomas : " expiation », "satisfaction », « mérite ». " Pour sauver ses élus, Dieu a envoyé Jésus-Christ pour satisfaire à sa justice, et pour mériter de sa miséricorde la grâce de rédemption, la grâce médicinale, la grâce de Jésus-Christ. » Par une décision de Dieu, le couronnement du « sacrifice continuel » que fut l'existence de Jésus se situe certes à la croix, mais plus encore au Jardin des oliviers, où - dit Pascal au Christ « vous suez le sang pour l'expiation de nos offenses ${ }^{22}$ ».

61

Cette théologie ne fait pas l'unanimité aujourd'hui. Elle présente deux graves défauts : elle donne au Christ la figure juridique d'un "substitut », et « la faute et sa réparation [y] restent extérieures l'un à l'autre : elles sont chacune sur un plateau de la balance ${ }^{23}$. » En second lieu, elle s'imagine Dieu comme un être qui réclame son dû et risque d'aboutir à un Dieu pervers, qui prend plaisir à la souffrance et ne saurait lui-même pardonner à ses ennemis, alors qu'il l'exige des hommes ${ }^{24}$. Les théologiens ne projettent-ils pas en Dieu une idée tout humaine de la «justice»? Alors que des paraboles comme celle des ouvriers de la onzième heure (Matthieu 20,1-16) ou celle de l'Enfant prodigue (Luc 15) nous mettent en garde contre cette naïveté. Le «Jugement dernier » ne fera-t-il pas que constater que certains hommes se sont exclus eux-mêmes de la Vie éternelle par leur refus de l'amour?

Devant les risques d'outrecuidance des théologiens, il faut rappeler fermement avec Urs von Balthasar que «les Chants du serviteur de Dieu [chez Isaie], Paul, Jean, les synoptiques [...] ont beau être éloquents et catégoriques sur le fait qu'ils affirment [la Passion et la mort de Jésus "pour nous »], ils n'en observent pas moins un silence respectueux sur le comment de ce transfert de la faute ${ }^{25} »$. Donc les comparaisons comme "sacrifice d'expiation » ou "rançon », utilisées spontanément par les premiers chrétiens venus du judaïsme et immergés dans une vieille culture sacrificielle, ne doivent pas égarer. Avec elles, ne sommes-nous pas à mille lieues du Dieu père de la magnifique parabole de l'enfant prodigue (Luc 15)?

Quoi qu'il en soit des incertitudes contemporaines, Pascal s'inscrit dans cette théologie de l'expiation. Nulle part il n'envisage que le pardon de Dieu n'ait été lié à l'Agonie et à la Passion que par une souveraine décision; que le «Il a fallu que le Christ souffrît » des évangiles ne renvoie qu'au Plan de salut sur le monde d'un Dieu révélant un mystère d'amour. Dans cette acceptation non problématique d'une vieille théologie, l'auteur des Pensées se comporte comme il l'a fait en recevant les constructions de saint Augustin sur la grâce en "paroles d'évangile ». Or, les théologies, si elles peuvent contribuer à maintenir le cœur du message chrétien, se révèlent provisoires et toujours approximatives. Mais cette prise de conscience du passage de l'histoire sur les formulations dogmatiques ne s'est développée qu'au XIX ${ }^{e}$ siècle. Fixiste, comme Bossuet, Pascal est ici un homme de son temps. 
64 Au terme de cette exploration, de quoi avons-nous pris plus clairement conscience? Tout d'abord de la prédilection de Pascal pour le mystère de l'Agonie. Il vérifie une expérience bien connue : chaque vrai chrétien reçoit de Dieu la grâce d'être "habité " par certains épisodes, ou par tel d'entre eux, de la trajectoire du Christ. À la fin du $\mathrm{XVII}^{\mathrm{e}}$ siècle, une Jeanne Guyon fut ainsi hantée par l'Enfance de Jésus ; au XX ${ }^{\mathrm{e}}$, le jésuite Teilhard de Chardin fut fasciné par la Transfiguration, qui inspira sa vision du rayonnement cosmique de Jésus sur le monde. Chez l'auteur du "Mystère ", l'abandon de Gethsémani se trouve en pleine harmonie avec sa permanente hantise de l'abandon, avec sa conviction que Dieu peut nous retirer sa grâce et nous laisser dans la déréliction à toute heure. On se rappelle que cette angoisse d'abandon règne au cœur du "Mémorial» (fr. 742) et que les dernières paroles intelligibles prononcées par l'agonisant furent : «Que Dieu ne m'abandonne jamais ${ }^{26}$ ! "

Cette importance de l'Agonie au Mont des oliviers explique que l'auteur du « Mystère » l'ait isolée de la Passion. Il la considère en elle-même, il s'y arrête. Il la perçoit comme dotée d'une singularité exceptionnelle, et il est vivement conscient de l'unicité et de l'intensité d'une souffrance tout intérieure, où le salut des hommes s'est joué. Cette crise est la plus parfaite manifestation du dessein d'amour de Dieu sur le monde.

Sa contemplation de Gethsémani, Pascal l'a nourrie à peu près exclusivement de textes que Port-Royal lui avait rendus chers. Nous découvrons ici à nouveau combien il est enraciné dans la vie du monastère.

67 Malgré ces lectures, l'originalité de la pensée saute aux yeux: qu'il s'agisse de l'interprétation d'« En agonie jusqu'à la fin du monde » ou de celle de la plainte inouie "Mon âme est triste jusqu'à la mort », du sens de "La face en terre » ou de "Dans l'incertitude de la volonté divine ", Pascal innove. Il ne craint pas de s'écarter de la vulgate thomiste, et l'on peut penser qu'il prend les versets de l'Agonie plus au sérieux que saint Thomas lui-même.

68 En dépit de sa reprise d'une théologie de la Rédemption controversée aujourd'hui, sa célébration du "Mystère de Jésus" apparaît dans son œuvre comme un centre d'irradiation.

\section{NOTES}

1. Dialogue de l'histoire et de l'âme charnelle, dans les Euvres en prose 1909-1914, Paris, Gallimard, Pléiade, 1961, p.450-469. L'épisode de Gethsémani est raconté par les trois évangiles synoptiques : Matthieu 26, 36-56 ; Marc 14, 38-50 ; Luc 22, 39-51. Jean ne mentionne que le lieu et l'arrivée de Judas : 18, 1-12.

2. Le premier paragraphe de la citation provient des Considérations sur les mystères de Jésus-Christ (manuscrit 110 de la Bibliothèque Mazarine), publiées seulement en 1882 ; mais Condren avait diffusé sa pensée en de nombreuses conférences et directions spirituelles, y compris à Port-Royal. En tout cas, ce paragraphe en dit long sur une doctrine que Pascal pouvait lire dans les Discours et lettres, par exemple dans l'édition de 1648, Paris, Sébastien Huré, p. 460-463 : tout le reste de la citation vient de la lettre 47 de cette édition; elle est adressée à une dirigée et intitulée : « Que les 
peines et agonies intérieures de l'âme sainte de Jésus nous doivent être en en grande révérence à l'honneur desquelles il faut porter les nôtres. »

3. « Méditation 59 ", citée par J.-C. de Nadaï, dans Jésus selon Pascal, Paris, Desclée, 2008, p. 294.

4. Voir Ph. Sellier, "Colorations oratoriennes" et "Pascal sacrifiant», dans Port-Royal et la littérature I Pascal, Paris, Champion, 2010. Dans cette étude, les références aux Pensées renverront à l'édition Sellier, Paris, Garnier, 2011.

5. Cuvres complètes de Pascal, éd. J. Mesnard, Paris, DDB, vol. II, 1970, p. 855.

6. Traduction Osty (Paris, Seuil, 1973), avec en note le renvoi à Gethsémani.

7. Cuvres complètes de Pascal, éd. citée, III, p. 289-290.

8. Voir la riche étude de l'exégète A. Feuillet, L'Agonie de Gethsémani, Paris, Gabalda, 1977. En épilogue, le père Feuillet s'est penché sur deux textes des Pensées, "Le Mystère de Jésus » (fr. 749) et le fr. 751, qu'il considère à tort comme formant une unité, selon les vues courantes à cette époque. Dans le fr. 751, les notations 16 (Jésus devenu «Ut immundus pro luto ", comme un homme souillé pour [toi qui n'est que] boue) et 19 ("tous vos fléaux sont tombés sur lui ») proviennent d'Isaïe 64, 6-8 et 53, 4-5. Ce dernier texte appartient au célèbre "Chant du Serviteur » souffrant, considéré par le Nouveau Testament comme une prophétie de la Passion. Comme Condren, le père Feuillet est conscient de la singularité de l'Agonie : «Certaines formes de la souffrance morale de Jésus sont propres à la scène du Mont des oliviers. » (p. 279).

9. Homélies sur l'évangile de saint Jean 44-54), éd. Berrouard, Paris, Bibliothèque augustinienne, vol. 73 B, Paris, Études augustiniennes, 1989 : «Quid est ergo quod turbat semetipsum Christus nisi ut significet tibi quomodo turbari tu debeas cum tanta mole peccati gravaris et premeris?»

10. Tetrateuchus : "Coepit contristari : quia non subito et praeter voluntatem, ut nobis accidit, sed sponte, quando, quomodo, quantum voluit, contristatus est. " D'autres augustiniens que Jansénius avaient repris l'analyse d'Augustin, comme Bérulle: de Jésus «souffrant de la part des juifs et des hommes " il distingue «Jésus souffrant de la part de lui-même. » (Euvres complètes, Paris, Cerf, 1995, vol. III, p. 81 et 249, citées par G. Descamps, " Du Mémorial au Mystère de Jésus ... », dans Pascal auteur spirituel, Paris, Champion, 2006, p. 224-225; mais il est douteux que Pascal ait lu Bérulle).

11. Chaque "Méditation" occupe à peu près une page. La quinzième suit fidèlement saint Thomas : "C'est ainsi que ce cher Jésus [...] abandonne la partie inférieure [de son âme] aux épouvantes des douleurs qu'elle devait endurer. » La seizième s'intitule : "Jésus pâlit de peur » et précise que tout cela se passe au lieu même où aura lieu le Jugement dernier. La vingt et unième illustre le soupçon de docétisme qu'on est tenté d'adresser à la conception thomiste de l'Agonie : "Excès de joie et de tristesse en l'âme de Jésus. Il n'appartient qu'à l'âme unie à un Suppôt divin, d'être capable tout à la fois d'une joie souveraine et d'une tristesse excessive ». Où trouver la moindre lueur de joie dans le "Mystère »? Seul le mot " ennui », auquel sont consacrées cinq «Méditations» (17 à 21) rencontre le verset «Jésus dans l'ennui » du poème pascalien; mais Chardon nous met en garde : cet ennui ne règne que dans la partie sensitive de l'âme. Rien de tel n'est suggéré ni par Pascal, ni dans les récits évangéliques de l'Agonie, que certains exégètes ont comparés à la terrible « Nuit » de divers mystiques (J. Lebreton, La Vie et l'enseignement de Notre Seigneur Jésus-Christ, Paris, Beauchesne, 1931, vol. II, p. 315-317 ; F. Dreyfus, Jésus savait-il qu'il était Dieu ?, Paris, Cerf, 1984, p.124-125).

12. Sur Pascal et Condren, voir Ph. Sellier, "Colorations oratoriennes ", art. cité dans la note 4. L'étude du père Stella, « Le Mystère de Jésus. Fonti et signicatività tipologica », a paru dans la revue Salesianum, 35 (1973), p.517-527. Elle renvoie à G. Busaeus, Manuel de méditations, Paris, 1650 (2 $2^{\mathrm{e}}$ partie, Sur la Passion).

13. «Pascal et la spiritualité des chartreux", dans la revue japonaise Équinoxe, 6 (1990), p. 5-20. Nous avons vu que Pascal lisait sous la plume de Condren, dans une lettre à une correspondante, que l'âme de Jésus dans l'Agonie était «toujours soucieuse de vous, plus pressée du souvenir de vos fautes. "

Courrier du Centre international Blaise Pascal, 37 | 2015 
14. Voir l'article remarquable de Pierre Pascal, «L'abbé de Saint-Cyran, les chartreux et les Solitaires ", réédité dans les Chroniques de Port-Royal, Paris, Vrin, nº57 (2007), p. 173-188. Condren avait été l'un des directeurs spirituels du monastère dans les années 1626-1633.

15. "Jésus-Christ chez Pascal ", dans Port-Royal et la littérature, I, Pascal, ouvrage cité, p. 510.

16. Somme contre les gentils, IV, 27, n.1.

17. Somme de théologie, IIIa pars, question 8. Toutes les références renverront à l'ample traité « Du Christ ", donc à la troisième partie de la Somme; les abréviations seront q. pour question, a. pour article, c. pour corps de l'article, $1 \mathrm{~m}$. pour réponse à la première objection, ad primum (et ainsi de suite).

18. J. Moltmann, Le Dieu crucifié, Paris, Cerf, 1980, p. 261. La théologie catholique classique, imprégnée de l'impassibilité attribuée à la divinité par les Grecs, peine à rendre compte de la Passion. Prend-elle assez au sérieux de nombreux textes bibliques, dont les récits de la Passion et les Lamentations de Jérémie, chantées dans la liturgie du Vendredi saint? En 1938, un thomiste déclaré comme Jacques Maritain s'interrogeait : «Le concept et le mot de douleur ne peuvent être employés à l'égard de Dieu que métaphoriquement [...] nous devons cependant chercher dans une perfection divine innominée l'exemplaire éternel de ce qu'est en nous la douleur avec sa noblesse. » ("Quelques réflexions sur le savoir théologique », dans la Revue thomiste, tome 69, $\mathrm{n}^{\circ} 1$ (1969), p. 23).

19. Cette interprétation est encore aujourd'hui celle de la Traduction œcuménique de la Bible (Matthieu 27, 46).

20. Somme de théologie, q.18, a.6, 3m.

21. Voir Maxime le confesseur, L'Agonie du Christ, Paris, Migne, «Les Pères dans la foi », 2010 et, dans les Pensées, le fr.347. Sur la connaissance par Pascal de l'hérésie monothélite, voir la XVII ${ }^{e}$ Provinciale, éd. Ferreyrolles, Paris, Garnier, 2010, p. 459 et sv.

22. Écrits sur la grâce, éd. citée, vol. III, p. 795 ; Prière pour demander à Dieu le bon usage des maladies, $\S 12$ : Ibid., IV, p. 1008. Sur l'existence du Christ comme «sacrifice continuel et sans interruption », voir la « Lettre sur la mort »(Ibid., II, 854) ; « Ce sacrifice a duré toute sa vie, et a été accompli par sa mort » (Ibid., II, 155).

23. H. Urs von Balthasar, "Le mystère de la substitution ", dans Je crois en un seul Dieu, Paris, P.U.F., coll. « Communio », 2005, p. 142.

24. Voir Paul Ricœur, "Entre philosophie et théologie I : la Règle d'Or en question", dans Lectures 3, Paris, Seuil, 1994, p. 273-279. Ricœur souligne que le Sermon sur la montagne appelle à une « économie du don ", à une « logique de la surabondance » qui se trouve au-delà de l'éthique ordinaire des hommes et de la « justice».

25. Op.cit., p. 142.

26. Je me permets de renvoyer à "Abandonné dans une île déserte ... Fantasmatique et théologie dans les Pensées », dans Essais sur l'imaginaire classique, Paris, Champion, 2005, p. 165-173.

\section{INDEX}

Mots-clés : Pascal, Christ, Gethsémani, Passion, Agonie

Keywords : Pascal, Christ, Gethsemane, Passion, Agony 
AUTEUR

PHILIPPE SELLIER

Professeur émérite de lettres

Université de Paris IV-Sorbonne 